\title{
SELF-CORRECTION IN APRAXIA OF SPEECH: THE EFFECT OF TREATMENT
}

\author{
Anita van der Merwe \\ Department of Communication Pathology \\ University of Pretoria \\ South Africa
}

Correspondence address:

Anita van der Merwe PhD

Professor

Department of Communication Pathology

University of Pretoria

Pretoria, 0002

SOUTH AFRICA

E-mail address: anita.vandermerwe@up.ac.za

Phone: $\quad 2712-4202357$

Fax: $\quad 2712-4203517$ 


\section{Self-correction in Apraxia of Speech: The effect of treatment}

Overt self-correction of incorrect speech production reflects an awareness of manifested speech errors. The outcome of production can be monitored by means of response-produced feedback of tactile-kinesthetic and auditory stimuli. However, selfcorrection may also occur before speech is executed. During speech motor planning the speaker can centrally monitor the efference (reference) copy of the planned utterance through internal feedback. For accurate production of the speech sound, comparison with an internal model of the motor plan in the sensorimotor memory may take place. Internal feedback may perform an error-correction function before speech is produced (Van der Merwe, 1997). The focus of this study is on self-correction of speech errors in the apraxic speaker.

Speech errors such as sound substitutions, sound distortions, distorted substitutions, slow speech rate and start-restart behavior are characteristic of apraxia of speech (AOS) (Duffy, 2005; McNeil, Robin \& Schmidt, 1997). The start-restart behavior may reflect the underlying disorder in speech motor planning, but also the attempt to produce error-free speech and self-correct manifested and upcoming speech errors. The questions addressed in this study relate to the effect of treatment on the number of overt self-corrections during the course of treatment, number of self-corrections as a percentage of the total number of incorrect productions, and successful and unsuccessful self-corrections as a percentage of the total number of self-corrections. It may be predicted that the number of self-corrections will decrease and the percentage of successful self-corrections will increase if speech improves (total number of incorrect productions decline) during treatment. It may also be predicted that the number of selfcorrections as a percentage of the total number of errors may decrease during treatment. A decrease in the percentage of self-corrections may indicate a shift from externally manifested (overt) self-corrections based on response-produced feedback to error correction of upcoming speech errors based on internal feedback. If internal predictive control (Kawato \& Gomi, 1992; Keele, 1982; Kelso, 1982; Van der Merwe, 1997) improves, the percentage of overt self-corrections may decline. Answers to these questions may contribute to a better understanding of speech motor control in AOS.

\section{Method}

The current study is part of a larger study on the outcomes of the Speech Motor Learning (SML) Program (Van der Merwe, 1985). The SML Program targets speech sound treatment in nonwords and words and incorporates motor learning principles. The previous study showed that the number of perceptual errors decreased during treatment (Van der Merwe, 1998; Van der Merwe, Tesner, Groenewald \& Moore, 1998). In the current study the data was further analyzed with regard to self-corrections. 


\section{Participant}

The participant was a university trained, bilingual, right handed male who suffered an embolic cerebro-vascular incident at the age of 52 years. The study started 30 months post-onset. The Boston Diagnostic Aphasia Examination (an informally translated Afrikaans version) showed no problems other than in fluency. The participant had normal hearing and no facial or tongue weakness. Radiological reports (MRI and CT) revealed small lesions near Broca's area and the left parietal-occipital and right occipital areas of the brain. He displayed speech signs typical of apraxia of speech (McNeil, et al., 1997).

\section{Procedures}

The SML Program (Van der Merwe, 1985) was applied for 18 months. The treatment was provided twice a week for an hour by the author. Three baseline tests (B1 to B3) were done before treatment commenced. Throughout the course of treatment, multiple tests (probes) were administered. The data of four of these tests, spanning the treatment period, were analyzed in the present study. Each word or nonword was printed on a separate card and presented to the participant one at a time. All productions were self-initiated in the sense that they were read. No time restriction was imposed. To provide the opportunity for self-correction, the instruction was to repeat each word and nonword three times consecutively.

\section{Materials}

The test stimuli consisted of 110 Afrikaans words and 110 nonwords that complied with the phonological rules of Afrikaans. Three repetitions of each of the 220 target utterances were scored for each baseline and each subsequent test. Test stimuli were not part of the treatment stimuli but were representative of the type of material used during treatment. The test stimuli were identical for all tests. The test stimuli contained 15 different consonants (C), 10 vowels (V) and eight consonant clusters in CVCV, CVC, CVCVC and CVCVCVC (or initial CC) syllable structures. The speech sounds represented easy and difficult sounds as perceived by the participant. All sounds except the consonant clusters had been treated.

\section{Perceptual analysis of self-correction}

Self-correction was judged perceptually by the author. These data were scored from tape recordings. The production of words and nonwords was regarded as incorrect when distortion, substitution, or distorted substitution of any speech sound occurred. The three repetitions of a target word or nonword were regarded as a single opportunity to self-correct production. To differentiate between audible trial-and-error groping (Duffy, 2005) and self-correction, self-correction was only judged as such if the client had said the entire word or nonword and then displayed a pause or start-restart behavior and attempts to change the utterance.

Production of a target utterance was scored as one of the following: (1) Three correct repetitions of the target utterance; (2) Wrong with no attempt to self-correct when one or more of the three repetitions were produced incorrectly with no pauses or startrestart behavior and no attempt to change production; (3) Successful self-correction when the incorrect production of the target word or nonword was followed by an attempt to 
correct a sound substitution, a sound distortion, or a distorted substitution; If a successful self-correction was followed by an error on the same target utterance during a next repetition, it was still accepted as an instance of successful self-correction. (4) Unsuccessful self-correction under same circumstances as successful.

\section{Reliability}

The rating system as developed by the author was verified by a second rater. Data from test B1 were analyzed by consensus to refine the formulation of the different ratings. The judgements made by consensus were then compared point-to-point to the ratings of the first rater. An agreement score of $85 \%$ was reached. The data of B1 were re-analyzed by the first rater and an intra-rater point-to-point agreement score of $87 \%$ was achieved.

\section{Results}

The number of incorrect productions of words and nonwords (see Figure 1) and the number of self-corrections (see Figure 2) decreased during treatment. However, selfcorrections as a percentage of the total number of incorrect productions remained almost similar during treatment (see Figure 3). The percentage of successful self-corrections (presented for words in Figure 4 and for nonwords in Figure 5) increased while the percentage of unsuccessful self-corrections decreased across time. Nonwords followed the same trend as words, but not as pronounced. A greater number of successful selfcorrections occurred on words than on nonwords.

\section{Discussion}

The decrease in number of incorrect productions and number of self-corrections together with the increase in the percentage of successful self-corrections seem to suggest that this client's speech production ability and overt self-correction improved during treatment. However, the almost unchanged number of self-corrections as a percentage of total number of incorrect productions suggests that prediction of upcoming errors did not improve. Speech errors continued to occur inadvertently and the mode of speech motor control seems unchanged. This result suggests that the process of internal predictive control remained dysfunctional in this apraxic speaker.

\section{References}

Duffy, J.R. (2005). Motor speech disorders: Substrates, differential diagnosis, and management. Missouri: Elsevier Mosby.

Kawato, M., \& Gomi, H. (1992). The cerebellum and VOR/OKR learning models. Trends in Neurosciences, 15, 445-453. 
Keele, S.W. (1982). Learning and control of coordinated motor patterns: The programming perspective. In J.A.S. Kelso (Ed.), Human motor behavior: An introduction. Hillside: Lawrence Erlbaum Associates.

Kelso, J.A.S. (1982). Concepts and issues in human behavior: Coming to grips with the jargon. In J.A.S. Kelso (Ed.), Human motor behavior: An introduction. Hillside: Lawrence Erlbaum Associates.

McNeil, M.R., Robin, D.A., \& Schmidt, R.A. (1997). Apraxia of speech: Definition, Differentiation, and treatment. In M.R. McNeil (Ed.), Clinical management of sensorimotor speech disorders. New York: Thieme Medical Publishers.

Van der Merwe, A. (1985). Treatment program for developmental apraxia of speech and other speech disorders (title translated). Publication of the University of Pretoria.

Van der Merwe, A. (1997). A theoretical framework for the characterization of pathological speech sensorimotor control. In M.R. McNeil (Ed.), Clinical management of sensorimotor speech disorders. New York: Thieme Medical Publishers.

Van der Merwe, A. (1998). Speech motor learning in apraxia of speech: Report on a single subject experimental study. Paper presented at the $8^{\text {th }}$ International Aphasia Rehabilitation Conference. Kwa Maritane, Pilanesberg National Park, S.A.

Van der Merwe, A., Tesner, H., Groenewald, E., \& Moore, C.A. (1998). Successive level intervention in apraxia of speech: Status report on a single subject experimental study. Paper presented at the Ninth Biennial Conference on Motor Speech: Motor Speech Disorders and Speech Motor Control, Tucson, Arizona, USA. 


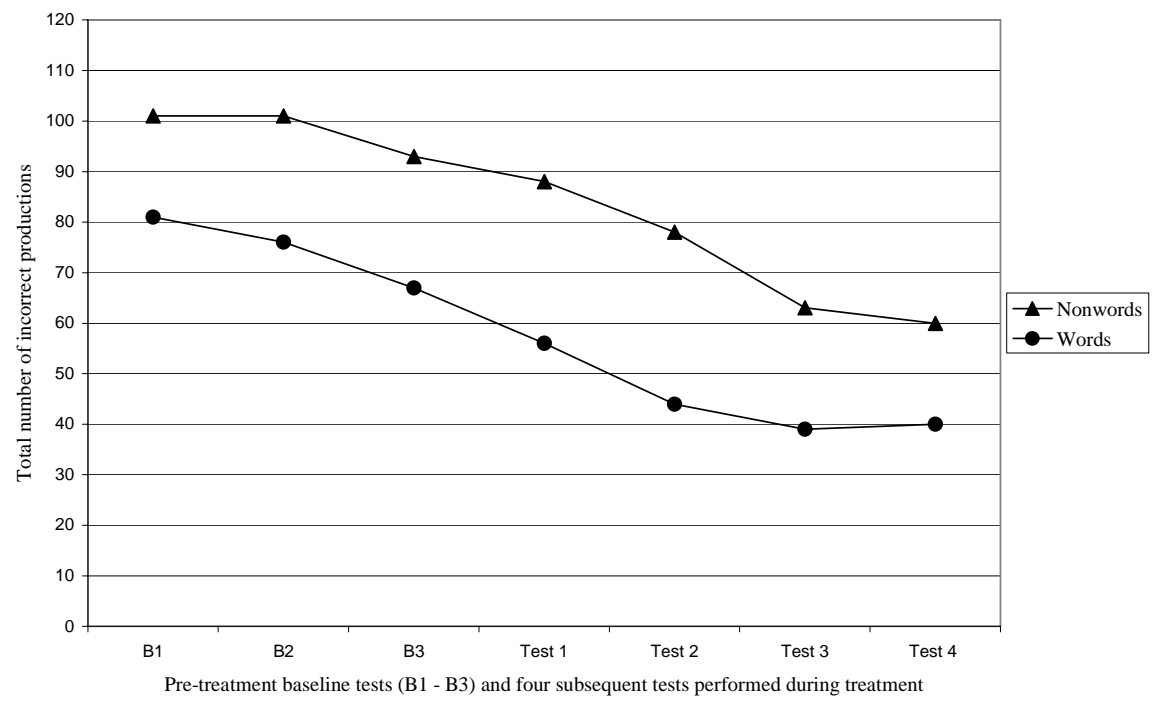

Figure 1: Total number of incorrect productions of nonwords and words 


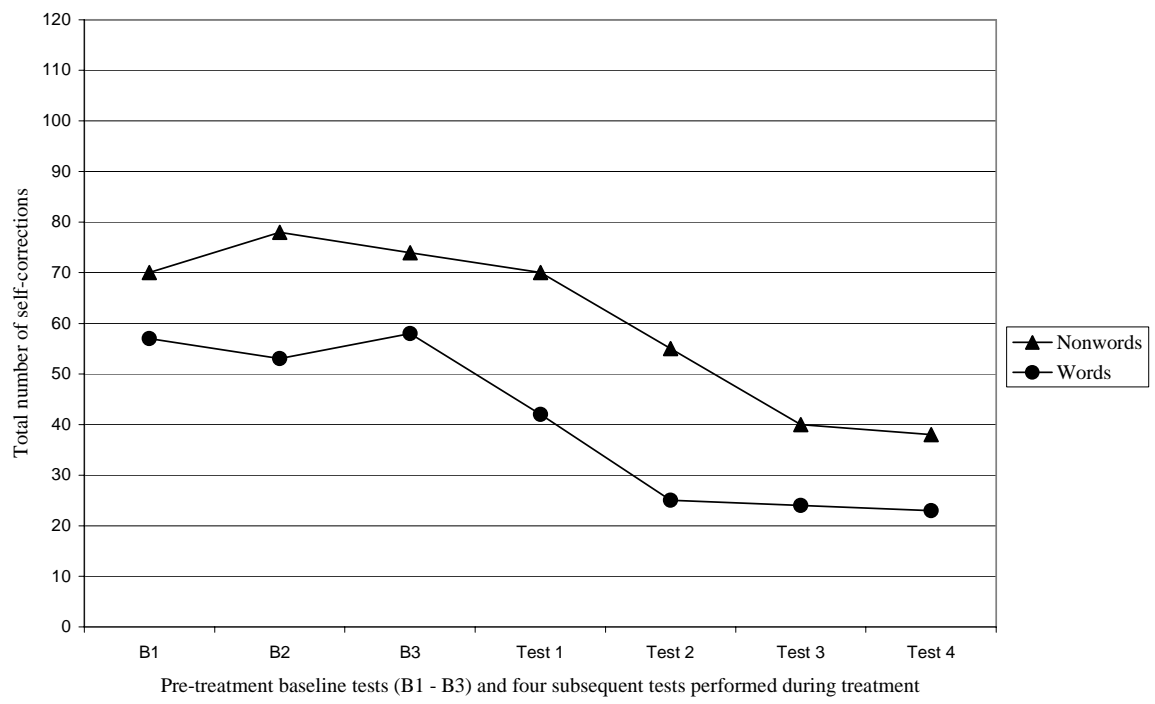

Figure 2: Total number of self-corrections on nonwords and words 


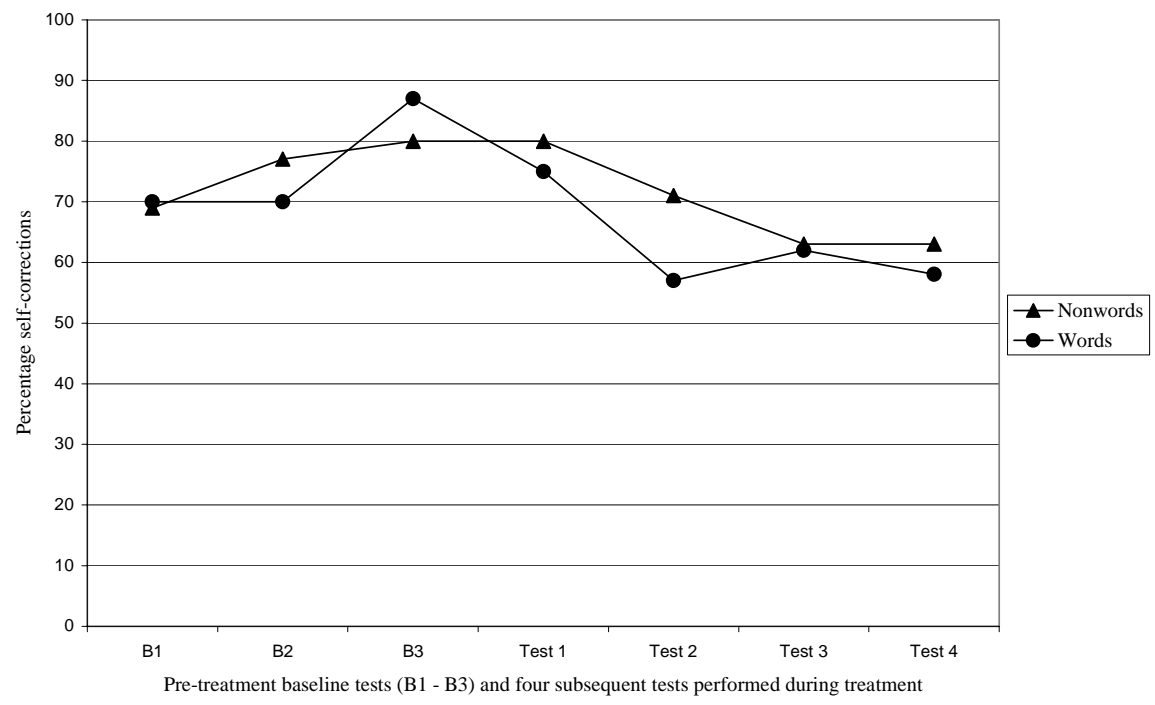

Figure 3: Self-corrections as a percentage of the total number of incorrect productions 


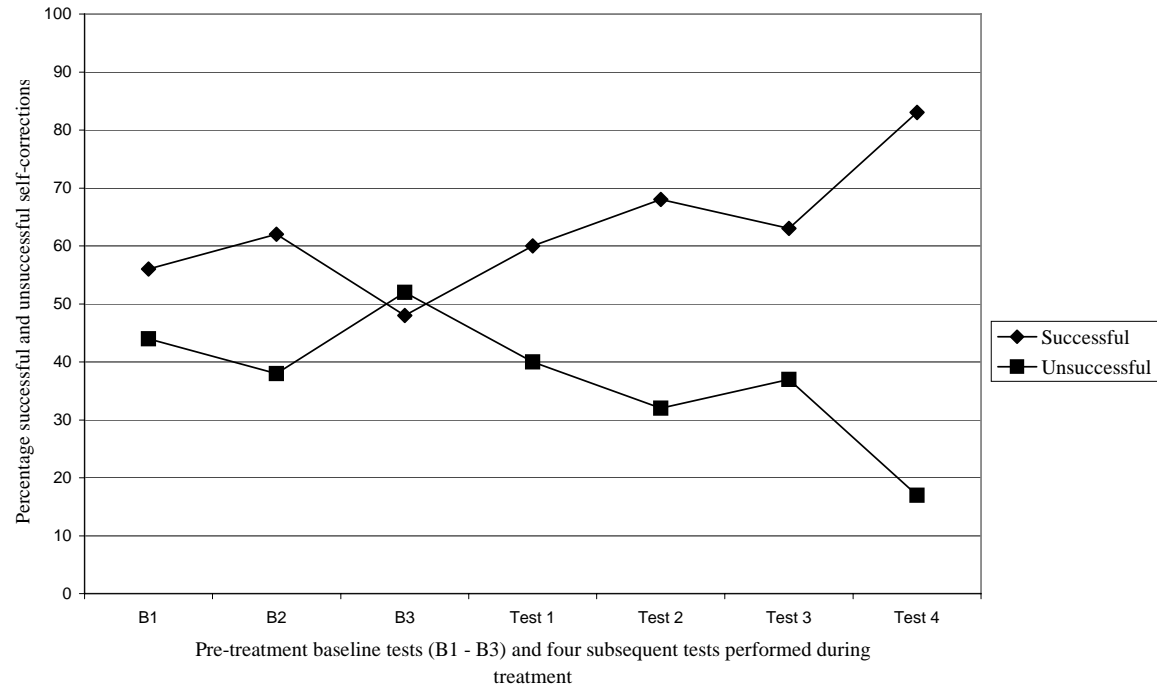

Figure 4: Successful and unsuccessful self-corrections of words as a percentage of the total number of self-corrections 


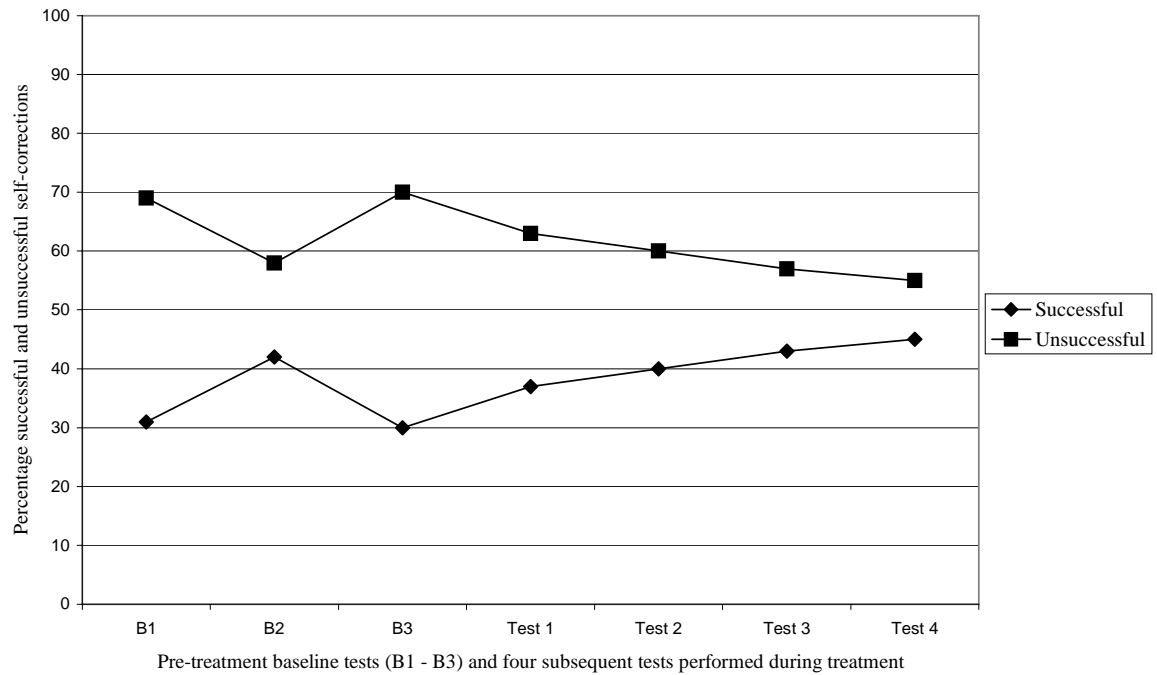

Figure 5: Successful and unsuccessful self-corrections of nonwords as a percentage of the total number of self-corrections 